\section{The probability of association between response inhibition and compulsive symptoms of obsessive-compulsive disorder: Response to Abramovitch and Abramowitz}

\section{To the Editors:}

Abramovitch and Abramowitz (2014) listed several problems with the conclusions they interpret as having been drawn from cross-sectional (correlational) data in our recent article (Harsányi et al., 2014). First of all, causal inferences such as response inhibition deficits underlying compulsive rituals cannot logically be concluded from correlational data. However, this conclusion was never drawn in our article.

Contrary to what Abramovitch and Abramowitz state in their Letter to the Editors, we did not conclude that response inhibition leads to obsessive-compulsive disorder (OCD); rather, we reported that inhibition deficit can be observed in OCD. We, of course, share the opinion of Abramovitch and Abramowitz that extensive deficits of executive functions, including response inhibition, are identifiable in several other psychiatric illnesses (e.g., in schizophrenia, bipolar affective disorder, attention deficit/hyperactivity disorder, anxiety disorders, and depression). We described correlations in our article based on the results of our research. However, we would like to emphasize the fact that in the case of the 51 OCD patients, we not only used questionnaires measuring executive functions (DEX, WBSI), but we also used a well-validated neurocognitive test battery to assess these functions.

Abramovitch and Abramowitz question whether the Dysexecutive Questionnaire (DEX) adequately measures executive deficits. They base their skepticism on a study by Chaytor et al. (2006) in which the correlation of the DEX with executive tests was not particularly high.

In reference, we would like to highlight that the study by Chaytor et al. (2006) is grounded on the data of a group of merely 46 patients, with a variety of neurological disorders, where correlation was carried out with a few tests measuring executive performance and experimental methods (Wisconsin Card Sorting Test (WCST), Trail Making Test, Stroop Color and Word Test, Controlled Oral Word Association Test (COWAT)), and between the DEX and other questionnaires. This study found a moderate correlation between the DEX and the Stroop task $(r=0.35)$ and weak or no correlation between the DEX and the Trail Making Test-Part B, COWAT and WCST $(r=0.25, r=0.28, \quad r=0.03$, respectively).

In our opinion, the biggest problem with this study is that it did not find a substantially stronger correlation between the other executive tests either. For example, the WCST shows an $r=0.34$

DOI of original article: http://dx.doi.org/10.1016/j.psychres.2014.01.050 correlation with the Trail Making Test-Part B, and an $r=0.33$ and $r=0.00$ correlation with the Stroop interference scores. Does this mean that the WCTS is also an inadequate tool for measuring executive deficit?

Wilson et al. (1996) and Burgess et al. (1998), in contrast with Chaytor et al. (2006), when standardizing the DEX, examined 92 patients with brain injuries (closed head injuries, encephalitis, dementia, stroke) and compared data from the DEX with one of the most comprehensive neuropsychological test batteries (Behavioral Assessment of the Dysexecutive Syndrome (BADS)), consisting of six sub-tests. Research data showed a strong correlation between the BADS total score and DEX other ratings $(r=-0.62)$.

The above-cited studies indicate that we can, indeed, regard the DEX score of significant other ratings as a reliable research tool. Since the manual of the DEX/BADS presents inclusive statistics of dysexecutive symptoms of frontal lobe brain injury patients, the sentence in our publication, namely that 'Based on the normative scales of the DEX questionnaire the presence and severity of executive problems of our 51 OCD patients were between 31 and 53 percentile, which shows a severe executive impairment comparing to the normal control of the manual of the DEX,' needs to be more accurate.

In the DEX/BADS manual, the percentage values are not representative of normal persons but of brain injury patients. Therefore, the fact that the executive function disorders in our examined OCD patients fell between 25 and 50 percent provides an even stronger basis for the presence of 'severe executive impairment', as it stands on a comparable level with the 'severe' extent of injury level reflecting executive deficit in brain injury patients. However, we did make an ambiguous statement in our article, but owing to the standardization logic of the DEX, the 2550 percent value in our examined OCD group (based on the data of a group of patients with brain injuries showing severe executive deficit) qualifies in the severely impaired range. Nonetheless, we can also assert, based on clinical data of our examined sample of patients, that DEX values correlated well with inhibition markers in the conducted executive tests measuring attentional focus and switching skills.

To summarize, in our opinion, it is far from clear that the DEX is an unreliable neuropsychological tool, based both on literature and our own data. The neuropsychological tasks used to examine executive functions in our research confirm that scores of the DEX other ratings are capable of describing executive deficit and provide an ecologically valid measurement.

Regarding OCD and impulsivity, on the one hand, we are of a similar opinion as Abramovitch and Abramowitz in that compulsions are "carefully planned and executed, and are usually carefully timed in response to obsessions.' At the same time, we disagree with their assertion based on clinical observations that '...in clinical work with patients, it is easy to observe that individuals 
with OCD are able to postpone or stop their rituals in certain circumstances (e.g., to avoid embarrassment, as part of behavior therapy), which indicates intact ability to inhibit these behaviors.' We hold that this clinical observation is also present in addictive behavior patterns (e.g., compulsive gambling) and in substance dependent addictive patterns (e.g., alcohol dependence) that essentially reflect disorders in impulse control, since in these clinical patterns patients are also able to suspend their impulsive behavior under humiliating circumstances. Furthermore, clinical tests verify the prevalence of impulsivity in OCD, as was recently reported in patients with $O C D$, with eating disorder (ED), and normal controls (Boisseau et al., 2012). They examined values of impulsivity and 'response inhibition' measured by cognitive tests on the above sample and found that 'Compared to controls, both clinical groups reported higher levels of impulsivity on the BIS-11 (Barratt Impulsivity Scale)[but] only the OCD demonstrated increased stop-signal reaction time. Heightened levels of selfreported impulsivity may reflect the experience of anxiety in both OCD and ED populations whereas a lack of inhibitory control may represent a specific behavioral deficit in OCD' (Boisseau et al., 2012).

Altogether, we wished to respond through the above remarks to the Letter to the Editors by Abramovitch and Abramowitz, but at the same time, we are delighted they have started a fruitful debate in these pages with their observations, which we hope will contribute to an increasingly accurate description of cognitive disorders accompanying OCD symptoms.

\section{References}

Abramovitch, A., Abramowitz, J.S., 2014. Improbability of response inhibition as a causal etiological factor of obsessive-compulsive disorder. Psychiatry Research 217 (3), 253-254.

Boisseau, C.L., Thompson-Brenner, H., Caldwell-Harris, C., Pratt, E., Farchione, T., Barlow, D.H., 2012. Behavioral and cognitive impulsivity in obsessivecompulsive disorder and eating disorders. Psychiatry Research 200, 1062-1066.

Burgess, P.W., Alderman, N., Evans, J., Emslie, H., Wilson, B.A., 1998. The ecologica validity of tests of executive function. Journal of the International Neuropsychological Society 4, 547-558.

Chaytor, N., Schmitter-Edgecombe, M., Burr, R., 2006. Improving the ecological validity of executive functioning assessment. Archives of Clinical Neuropsychology 21, 217-227.

Harsányi, A., Csigó, K., Rajkai, C., Demeter, G., Németh, A., Racsmány, M., 2014. Two types of impairments in OCD: obsessions, as problems of thought suppression; compulsions, as behavioral-executive impairment. Psychiatry Research 215 (3), 651-658.

Wilson, B.A., Alderman, N., Burgess, P.B., Emslie, H., Evans, J.J., 1996. Behavioural Assessment of the Dysexecutive Syndrome (BADS). Thames Valley Test Company.

András Harsányi*, Katalin Csigó, Csaba Rajkai, Attila Németh Nyíró Gyula Hospital, II Psychiatric Department, Lehel út 59,

1135 Budapest, Hungary

E-mail address: Harsanyia@hotmail.com (A. Harsányi)

Gyula Demeter, Péter Pajkossy, Mihály Racsmány

Department of Cognitive Science, Budapest University of Technology and Economics, Stoczek utca 2, 1111 Budapest, Hungary

Received 14 March 2014 